\title{
Photoacoustic molecular imaging using combined acupuncture and gold nanorods as a composite contrast agent
}

\author{
Dan $\mathrm{Wu}^{*, \dagger}$, Xinxin Zhang ${ }^{*, \dagger}$, Jian Rong ${ }^{\dagger, *}$ and Huabei Jiang* ${ }^{*, \dagger, \S, \uparrow}$ \\ *School of Electronic Science and Engineering \\ University of Electronic Science and Technology of China \\ Chengdu, Sichuan, P. R. China \\ ${ }^{\dagger}$ Center for Information in Biomedicine \\ University of Electronic Science and Technology of China \\ Sichuan, P. R. China \\ ${ }_{\text {School of Physics }}$ \\ University of Electronic Science and Technology of China \\ Chengdu, Sichuan, P. R. China \\ ${ }^{\S}$ Department of Medical Engineering \\ University of South Florida \\ Tampa, FL 33620, USA \\ Ihjiang1@usf.edu
}

Received 7 October 2018

Accepted 3 May 2019

Published 23 May 2019

\begin{abstract}
In this study, we developed a novel photoacoustic imaging technique based on poly (ethyleneglycol)-coated (PEGylated) gold nanorods (PEG-GNRs) (as the contrast agent) combined with traditional Chinese medicine (TCM) acupuncture (as the auxiliary method) for quantitatively monitoring contrast enhancement in the vasculature of a mouse brain in vivo. This study takes advantage of the strong near-infrared absorption (peak at $\sim 700 \mathrm{~nm}$ ) of GNRs and the ability to adjust the hemodynamics of acupuncture. Experimental results show that photoacoustic tomography (PAT) successfully reveals the optical absorption variation of the vasculature of the mouse brain in response to intravenous administration of GNRs and acupuncture at the Zusanli acupoint (ST36) both individually and combined. The quantitative measurement of contrast enhancement indicates that the composite contrast agents (integration of acupuncture and GNRs) would greatly enhance the photoacoustic imaging contrast. The quantitative results also have the potential to estimate the local concentration of GNRs and even the real-time effects of acupuncture.
\end{abstract}

Keywords: Photoacoustic tomography; acupuncture; contrast agent; nanorods; Zusanli acupoint.

\footnotetext{
\Corresponding author.
}

This is an Open Access article published by World Scientific Publishing Company. It is distributed under the terms of the Creative Commons Attribution 4.0 (CC-BY) License. Further distribution of this work is permitted, provided the original work is properly cited. 


\section{Introduction}

Photoacoustic tomography (PAT), an emerging biomedical imaging technique, has developed greatly in recent decades. ${ }^{1-7}$ This in vivo brain functional imaging technique has the following unique advantages: (1) it is an noninvasive imaging mode which is conducted in accordance with ethical principles; (2) animals can maintain normal physiological activity during imaging; (3) it has adequate spatial resolution (up to tens of $\mu \mathrm{m}$ ), temporary resolution (up to tens of ms) and limited depth of penetration (up to several $\mathrm{mm}$, with intact scalp) in monitoring the brain; (4) it provides high-resolution functional information, such as hemoglobin and blood oxygenation levels relevant to describing physiology and pathology. ${ }^{6,8-20}$ PAT of small animal brain has successfully been implemented..$^{3,8-13}$ In these studies, PAT was used for mapping the microvascular network of a mouse brain with the hemodynamic activities when the scalp and skull were intact. ${ }^{8,10-13}$ Resting-state functional connectivity (RSFC) has been studied in the mouse brain using PAT. ${ }^{8,11}$ PAT was able to detect connectivity between different functional regions and even between sub-regions. ${ }^{8,10}$ Recently, several research demonstrated that the neonatal brain imaging through fontanel using photoacoustic technique is feasible. ${ }^{14-20}$ Even though the skull and scalp of any small animal is thin, the penetration depth is limited. ${ }^{17,20}$ In the situation where a limited detection depth is certain, or an endogenous contrast is not available, an exogenous contrast agent can be utilized. Oral or injectable chemicals, known as exogenous contrast agents, can be used to enhance images in medical scans of the tissues or organs. Besides enhancing the contrast, they can also potentially improve the image resolutions. ${ }^{4}$

Noble metal nanoparticles have been widely used as contrast agents in PAT due to their intrinsic optical absorption, surface plasmon resonance (SPR), effective surface modification, and high technical maturity of preparation. The optical properties of gold nanoparticles can be readily tuned by varying their size and shape. ${ }^{21-26}$ Gold nanorods (GNRs) are yet another example of an excellent photoacoustic contrast agent. They are able to display optical cross-sections comparable to nanospheres and nanoshells, however, at a much smaller effective size. Their optical resonance can be linearly tuned across the near-infrared region by changing either the effective size or aspect ratio of the nanorods. GNRs show per micron absorption and scattering coefficients that are an order of magnitude higher than those of nanoshells and nanospheres. ${ }^{26}$ Wang et al., developed a photoacoustic imaging technique based on GNR for quantitatively monitoring a focused-ultrasound (FUS) induced blood-brain barrier (BBB) opening in a rat model in vivo. Their experimental results showed that GNR contrast-enhanced photoacoustic microscopy (PAM) successfully revealed the spatial distribution and temporal response of the $\mathrm{BBB}$ disruption area in the rat brains. ${ }^{23}$ In addition, $\mathrm{Li}$ et al., conducted studies involving HER2, EGFR, and CXCR4 as the primary target molecules binding to GNRs in order to examine two types of cancer cells, OECM1 and Cal27. OECM1 cells overexpressed HER2, but exhibited a low expression of EGFR, whereas Cal27 cells showed the opposite expression profile. Single and double targeting resulted in signal enhancements of up to $3 \mathrm{~dB}$ and up to $5 \mathrm{~dB}$, respectively, and therefore portrayed a potential to improve cancer diagnoses. ${ }^{24}$

Traditional Chinese medicine (TCM) acupuncture involves inserting stainless steel needles into the skin at specific points on the body, called acupoints, so that a disease can be treated. Acupoints are distributed along meridians and collaterals, also called jingluo in traditional Chinese medicine, which is a system of internal main and collateral channels, regarded as a network of energy passages. ${ }^{27-30}$ An extensive body of research has demonstrated that the regulation effect of acupuncture and moxibustion at the acupoints on humans and animals is closely related to the effect and adjusting function of the stimulation on the nervous system. ${ }^{27-41}$ Acupuncture at the peripheral nervous system can exert a different influence on the central nervous system including the brain stem, hypothalamus, basal ganglia and cerebral cortex. ${ }^{39}$ Previous research in our laboratory has shown that in acupuncture, the Yongquan (KI1) and the Yanglingquan (GB34) acupoints can noninvasively increase the hemodynamics, including the cerebral blood volume (CBV) and total hemoglobin ( $\mathrm{HbT})$ in the in vivo mouse brain. ${ }^{31-34}$

Here, we considered poly(ethyleneglycol)-coated (PEGylated) gold nanorods (PEG-GNRs) as the photoacoustic contrast agent and considered acupuncture as a physical auxiliary method to adjust the hemodynamics of the mouse brain. In the study, 
we developed a method based on combined acupuncture and GNRs (as contrast agents), in which GNRs were injected intravenously and the concentration of hemoglobin in the cerebral cortex of the mice was increased by acupuncture at the Zusanli (ST36) acupoint. In our work, PAT was utilized to obtain images of the in vivo mouse brain over time, and to monitor the changes in the optical absorption of living mouse brain vasculature in response to GNRs injection and acupuncture both individually and together.

\section{Materials and Methods}

\subsection{Experimental animals}

22 Male specific pathogen free (SPF) Institute of Cancer Research (ICR) mice, weighing $28 \pm 5 \mathrm{~g}$, were used in this study. All animal experiments were approved by our Animal Ethics Committee. Mice were anesthetized with $10 \%$ chloral hydrate solution, which was injected intraperitoneally to the animals with a dosing of $4 \mathrm{ml} / \mathrm{kg}$ body weight. A constant temperature system was used to help maintain body temperature at $37 \pm 1^{\circ} \mathrm{C}$, which is crucial to lowering the death rate during the experiment. Before imaging, the hair on the mouse head, neck and left hind limb was removed with electric hair clippers and followed with depilatory cream. As required, we marked the ST36 acupoint, which is located lateral and inferior to the knee joint, $3.5 \mathrm{~mm}$ below the capitula fibula. During imaging, the mice were placed in a custom fixation device where their head, tail and limbs were fixated. Ultrasonic coupling agent was applied on the mouse head for coupling to the polyethylene film above to help maximize ultrasound transmission.

\subsection{Photoacoustic tomography set-up}

A custom circular-scanning PAT system is used in this study. In this system (see Fig. 1), a OPO laser pumped by a Q-switched Nd:YAG laser (Surelite I20, Continuum, CA, USA) operating at $700 \mathrm{~nm}$ with a pulsed duration of $10 \mathrm{~ns}$, and a repetition rate of $10 \mathrm{~Hz}$ served as the excitation source. In this work, the pulsed light power was about $8 \mathrm{~mJ} / \mathrm{cm}^{2}$ at the surface of the animal's head, which satisfies the laser security condition of permissible exposure for skin. The mouse in the holder was placed on a jack with adjustable height. Below the laser was a water

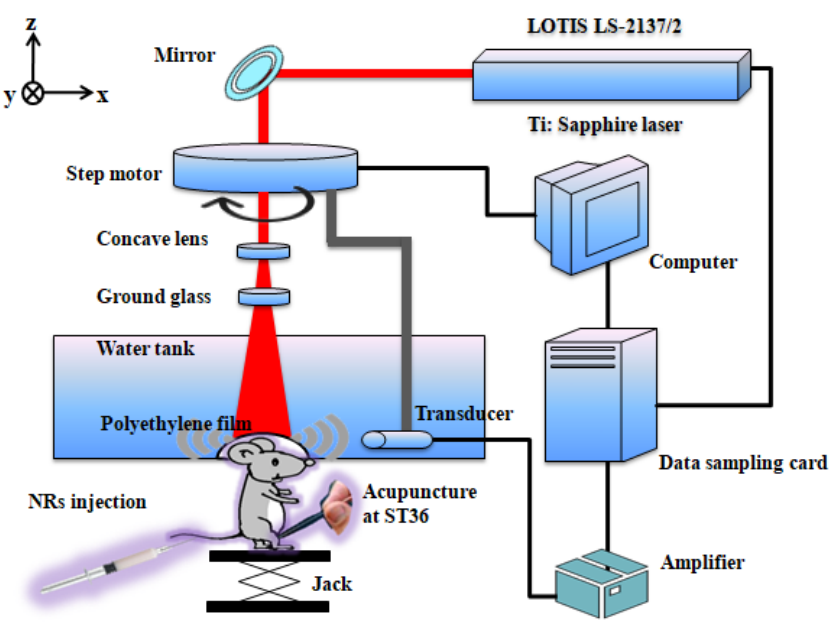

Fig. 1. Schematic of the home-made PAT system for in vivo mouse brain imaging, employing the GNRs contrast agent or acupuncture at ST36.

tank with an opening at the bottom center which is sealed with diaphanous polyethylene film. The animal head coated with a coupling agent and was kept close to the polyethylene film. The laser beam is expanded by a concave lens and then uniformly distributed by a pane of ground glass. The mouse head absorbs light energy after being irradiated by the emergent light and produces photoacoustic signals which are detected by a $5 \mathrm{MHz}$ nominal frequency transducer kept about $50 \mathrm{~mm}$ from the top of the head in the water. Rotated by a step motor, the transducer makes a $360^{\circ}$ turn centered on the mouse head. Thus, signals at 180 locations are detected in one imaging experiment. The scanning time for each frame of imaging was about $20 \mathrm{~s}$. PAT brain images are reconstructed from the photoacoustic signals using the delay and sum reconstruction algorithm.

\subsection{Contrast agents: PEG-GNRs}

Exogenous contrast agents used in PAT are able to greatly enhance the imaging contrast and potentially break through the imaging depth limit. Additionally, the agents could also be used for therapy in conjugation with drugs or in photothermal therapy, where it functions as an enhancer for the integration of diagnosis and therapy. Some contrast agents conjugated with a molecular probe (such as antibodies, proteins, nucleic acids and peptides) possess an active-targeting ability. As ligands, they bind to a specific subset of receptors in target cells or tissues. ${ }^{4}$ Other contrast agents (typically $60-400 \mathrm{~nm}$ 
diameter) have a passive-targeting ability, that is, they can extravasate into tumor tissues because of impaired vasculature and the enhanced permeability and retention (EPR) effect in solid tumors, which has been attributed to dysfunctional anatomical conditions such as localized leaky circulatory and lymphatic systems. ${ }^{42-52}$ For example, plasmonic noble metal nanoparticles, quantum dot (QD), carbon nanotubes and iron oxide nanoparticles have a passive-targeting ability. ${ }^{13,53-67}$ Their small size enhances transmigration across the blood-brain barrier (BBB), which protects the brain from harmful substances but also prevents the effective delivery of contrast agents. ${ }^{13}$ As a result, nanoparticles can accumulate in the vicinity of the brain vasculature and enhance optical contrast. Hanada et al., evaluated the permeability of NPs through the $\mathrm{BBB}$ using a cell-based in vitro $\mathrm{BBB}$ model. The $30 \mathrm{~nm}$ nanoparticles, especially at the high concentration, were transported thorough the BBB model, mirroring the same result reported in an animal model. ${ }^{57}$

In this study, we used PEG-GNRs as contrast agents for the in vivo brain photoacoustic imaging. PEG-GNRs were synthesized by Sheng Taier
Biomedical Science \& Technology Co., Ltd. By tuning the averaged dimensions of AuNRs to $30 \mathrm{~nm}$ by $10 \mathrm{~nm}$, their absorption peak was shifted to $675 \mathrm{~nm}$ wavelength. The thickness of the PEG coat was about $5 \mathrm{~nm}$. Scanning electron microscope (SEM) images showed that the nanoparticle solution in our laboratory had 10 by $30 \mathrm{~nm}$ PEG-GNRs (see Fig. 2(a)). Figure 2(b) describes the optical absorption spectrum of these GNRs. As shown in the optical absorption spectrum of these gold nanoparticles, we found that they exhibited a strong plasma resonance peak around $700 \mathrm{~nm}$. Phantom experiments were conducted to compare the optical absorption between GNRs and the mouse blood. Firstly, two tubes $(0.2 \mathrm{ml}, 3 \mathrm{~mm}$ in diameter) loaded with a mixture of GNRs and blood (tube 1), and blood (tube 2) were respectively embedded in a phantom consisting of agar powder $(2 \%)$. Anticoagulants are added to the blood in both tubes in order to keep the blood from clotting. The concentration of the nanoparticle solution was $3.75 \times 10^{11} \mathrm{GNRs} / \mathrm{ml}$. $0.05 \mathrm{ml}$ of nanoparticle solution and $0.15 \mathrm{ml}$ of blood was mixed into tube 1 and $0.2 \mathrm{ml}$ of blood was loaded into tube 2 . We then immersed the solid phantom into the water tank.

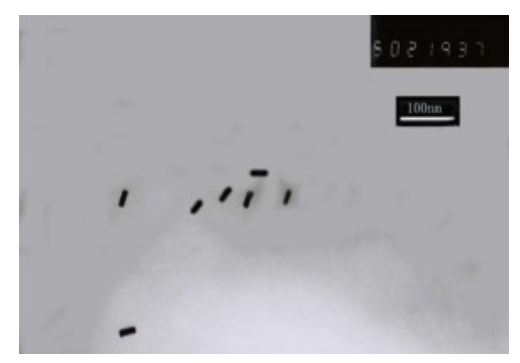

(a)

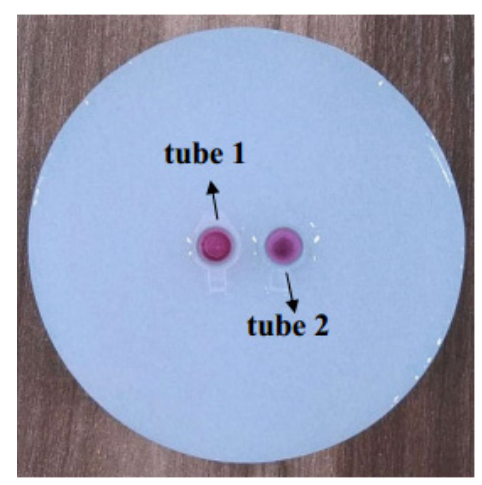

(c)

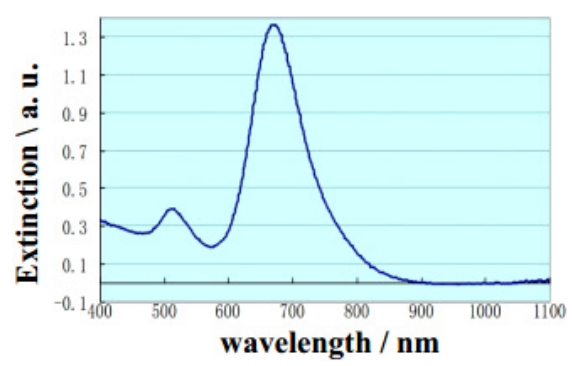

(b)

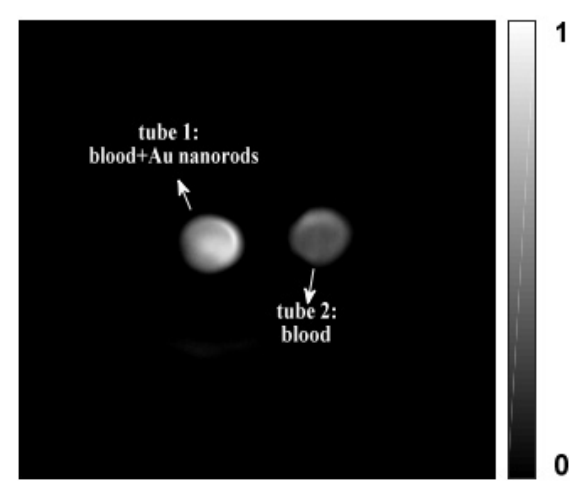

(d)

Fig. 2. (a) SEM image of the GNRs (Au nanorods) used in our study. (b) Optical absorption spectrum of these GNRs. (c) Photograph of tubes 1 and 2 embedded in a phantom. (d) Photoacoustic image for tubes 1 and 2. 
Figures 2(c) and 2(d) present the reconstructed images of the two objects at $700 \mathrm{~nm}$ and the photograph of the cross section of the phantom, respectively. Figure 2(d) shows a higher optical contrast of tube 1 than that of tube 2 . The obtained results indicated that GNRs would be able to display contrast enhancement in the vasculature by PAT, at a low dose.

When GNRs solution were added to the blood, the internal light absorption coefficient, which was originally determined by the endogenous contrast, such as hemoglobin in the blood, was further enhanced due to the absorption of gold nanoparticles. Functional light absorption coefficient changes in the GNRs injected tissue (i.e., RCBV) were constructed according to the following equation:

$$
\mu_{a}^{T}=\sigma_{a}^{\mathrm{HbT}} C_{\mathrm{HbT}}+\sigma_{a}^{\mathrm{GNR}} C_{\mathrm{GNR}},
$$

where the internal absorption provided by the blood is equal to the product of the extinction coefficient $\left(\sigma_{a}^{\mathrm{HbT}}\right)$ of the total hemoglobin and its concentration $\left(C_{\mathrm{HbT}}\right)$. The exogenous absorption provided by GNR is equal to the product of extinction coefficient $\left(\sigma_{a}^{\mathrm{GNR}}\right)$ of the contrast agent and its concentration $\left(C_{\mathrm{GNR}}\right)$ in the vascular system. Equation (1) shows that optical absorption in the vasculature is proportional to the concentration of total hemoglobin and GNR.

\subsection{Acupoint selection}

Many studies have shown that there is a relationship between acupoints and functional brain areas. ${ }^{27-41}$ The ST36 point is located laterally and inferiorly to the knee joint, $3.5 \mathrm{~mm}$ below the capitula fibula. There are many acupoints including the ST36 point in the circulation of Foot-Yangming stomach meridian. Numerous studies have shown that acupuncturing at the ST36 acupoint has proved effective in the prophylaxis and therapy of gastrointestinal diseases, insomnia, insanity, dizziness, and acute stroke. ${ }^{37-41}$ Additionally, previous and recent studies in our lab have applied PAM to investigate brain hemodynamic changes in response to electronic acupuncture (EA) at ST36. Six healthy mice were stimulated at the acupoint and three healthy mice were stimulated at sham points. Remarkable CBV changes in sensorimotor and retrosplenial agranular cortex were observed. Results showed the potential of PAM as a visualization tool to study the acupuncture effect on brain hemodynamics in animal models. ${ }^{31-34}$ In this study, we chose the ST36 point in the mouse's left hind limb as the stimulated acupoint. Previous comparisons have shown that there was no significant difference between acupuncture the left point and right point in previous studies. ${ }^{31}$

\subsection{Experimental protocols}

To evaluate the degree of PEG-GNRs delivery in the mouse brain, we designed three groups of PAT subjects. In each group, mice were anesthetized by chloral hydrate first, then shaved, marked at the acupoint and fixated before photoacoustic imaging. The entire preparation process took about $20 \mathrm{~min}$. We took a frame of imaging every $4 \mathrm{~min}$. Every mouse was exposed to the same experimental environment. For every mouse in the first group (Group I, $n=8), 10$ PAT images were taken. Every mouse was injected twice in succession with PEG-GNRs solution, each at about $6 \times 10^{8} \mathrm{GNRs} / \mathrm{g}$ body weight, in the caudal veins. One image was taken before injection and nine other images were taken after injection. Each mouse in the second group (Group II, $n=8$ ) was acupunctured at the ST36 point. One image was taken before acupuncture and seven other images were taken after acupuncture. Each mouse in the third group (Group III, $n=6$ ) was first injected with the GNRs solution and acupunctured at the ST36 point. The 1st image was taken before GNRs injection, the 2nd image was taken before the acupuncture and seven other images were taken after acupuncture. After photoacoustic imaging, all the mice were euthanized by an overdose of chloral hydrate.

\subsection{Statistical analysis}

The experiment was designed to quantitatively measure contrast enhancement and the corresponding optical absorption changes. All values are shown as means \pm standard error of mean (SEM). Statistical analysis was performed using unpaired $t$-tests. Statistical significance was defined as a probability $(p)$ value of $<0.05$. Analysis of variance (ANOVA) was performed to assess the changes of optical absorption and coherences in different stimulation and between cases using the ANOVA with Fisher's least significant difference post hoc 
analysis. All statistical analyses were performed with GraphPad Prism (version 5.0, GraphPad Software, USA). The data is presented as the means \pm standard deviation.

\section{Results and Discussion}

More than one external stimulation was applied in the work, which means multiple factors should be considered. Firstly, previous research in our laboratory has shown what the real change of cerebral hemodynamic parameters were in the normal physiological conditions during experiments without external stimulation. The previous result indicated that the normalized relative optical contrast presented a slightly dynamic downward trend authentically, which shows that even if the external stimulation was not performed, the phenomenon of declined optical absorption would occur. ${ }^{31-34,41}$

\subsection{Effect of PEG-GNRs injection on brain imaging}

Secondly, we need to make clear whether the contrast enhancement was induced by either the contrast agent or acupuncture separately. We then give the in vivo photoacoustic images for Group I $(n=8)$, where nanoparticles administration was implemented separately. Figure 3 present PAT brain images of a mouse from Group I. Ten minutes prior to the imaging, the mice were anesthetized and fixated. The extent of the superficial cerebrovascular distribution in the PAT image (Figs. $3(\mathrm{~b})-3(\mathrm{k})$ ) is in agreement with the open-scalp anatomical photograph (Fig. 3(a)). As shown in Figs. 3(b)-3(k), photoacoustic images were acquired at 10 time points for each mouse, i.e., prior to injection (Fig. 3(b)), and $4 \mathrm{~min}$ to $36 \mathrm{~min}$ post injection (Figs. $3(\mathrm{c})-3(\mathrm{k})$ ). Each mouse was administered successively with the GNRs solution twice, and two shots were taken at an interval of several seconds. Each mouse can only receive an injection of $0.2 \mathrm{ml}$ per shot. The major blood vessels shown in the PAT image (Fig. 3(a)) were marked by anatomical landmarks, including the rostral rhinal vein (RRV; the left RRV and right RRV was marked by No. 1 and No. 2 ellipses in red, respectively), the superior sagittal sinus (SSS, between the left and right hemispheres), the transverse sinus (TS, between the colliculi and cerebellum areas; the left TS and right TS was marked by No. 3 and No. 4 ellipses in red, respectively), the left veins (LVs), and the right veins (RVs). With the injection of the PEG-GNRs, the optical contrast of the brain vasculature became noticeably enhanced. The experimental timeline for Group I is shown in Fig. 3(l). Compared to the image prior to injection, the images post injection show the brain vasculature with greater clarity. Furthermore, the contrast agent lighted some "hidden" vessels (which were not visible due to their low contrast in the image prior to injection, i.e., the left RRV and right RRV) after the nanoparticles injection. It can be seen that there is an optical contrast peak in the mouse brain from 4 min to $8 \mathrm{~min}$ after injection. The results indicate that part of the contrast agents have been delivered effectively to the mouse brain, which results in enhancing the contrast of the brain. The absorption slowly declined after reaching the peak, but remained greater than the initial state absorption until the end of the image acquisition because of the clearance of the nanoparticles by the BBB. Besides, not all of the brain areas have increased optical contrast after the GNRs was injected. It suggests that contrast agents cannot penetrate all of the brain areas. $\mathrm{HbT}$ in the region of interest (ROI, including SSS) was measured in the discussion section. The color bars of the images were scaled in an uniform optical contrast (normalization).

\section{2. $\quad$ Effect of acupuncture at the ST36 point on brain imaging}

Thirdly, we look at the in vivo photoacoustic images for Group II $(n=8)$, where acupuncture at the ST36 point in the left side was implemented separately. Previous research in our laboratory has found that the acupuncture, either on the right or left points, would approximately enhance the contrast and increase the $\mathrm{HbT}$ in the two hemispheres. ${ }^{31}$ Figure 4 presents PAT brain images of a mouse from Group II. As shown in Figs. 4(a)-4(h), photoacoustic images were acquired at eight time points for each mouse, i.e., prior to acupuncture (Fig. 3(a)), and $4 \mathrm{~min}$ to $28 \mathrm{~min}$ post acupuncture (Figs. 4(b)-4(h)). We quickly inserted the sterile stainless-steel needle (diameter: $0.25 \mathrm{~mm}$; length: $25 \mathrm{~mm}$ ) vertically into the skin at the ST36 point, with a depth of 3 to $4 \mathrm{~mm}$. Then we manually twisted the needle quickly at a constant frequency 


\section{Group I : GNRs Group}

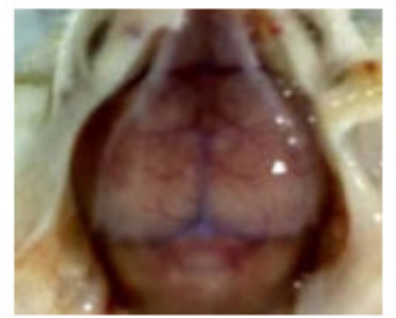

(a)

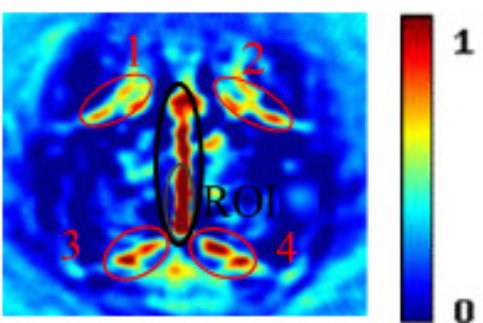

(b)

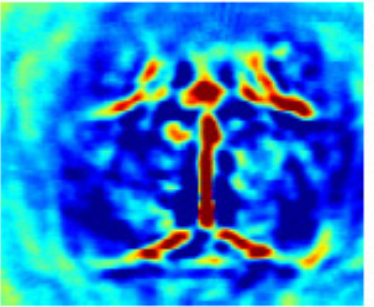

(c)

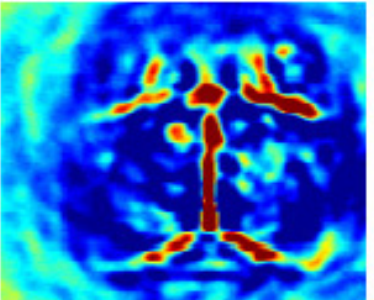

(f)

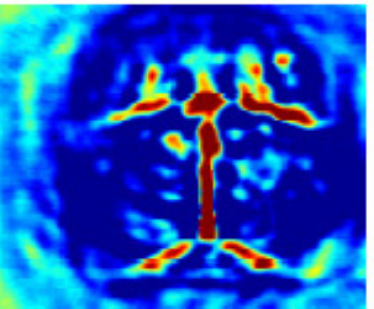

(i)

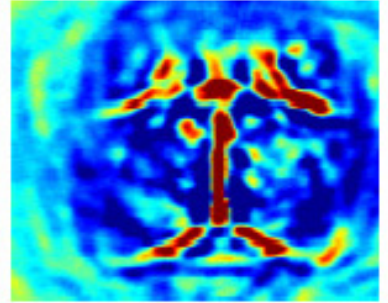

(d)

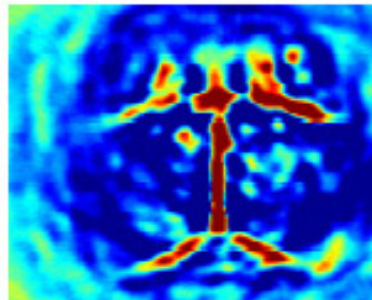

(g)

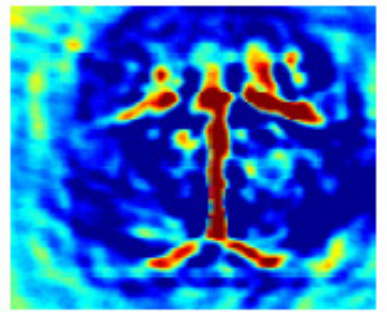

(j)

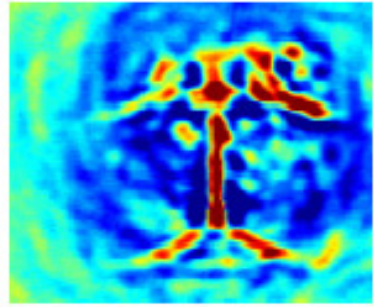

(e)

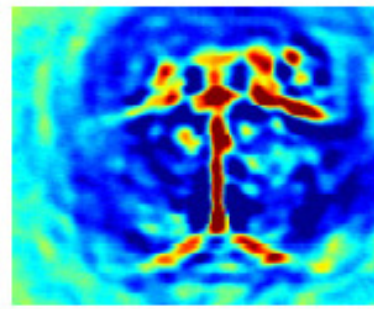

(h)

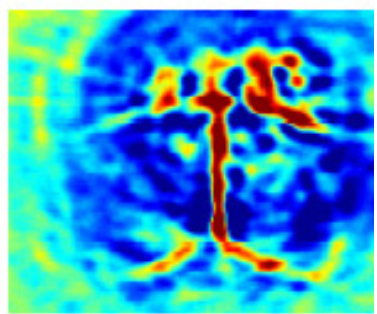

(k)

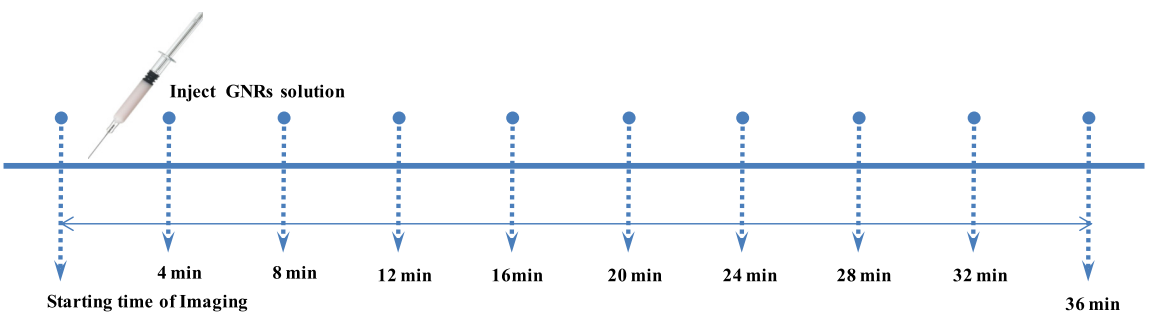

(1)

Fig. 3. (a) Open-skull photograph of a mouse brain surface after the imaging experiments. (b)-(k) 10 in vivo PAT brain reconstructed images of the cerebral vasculature of a mouse brain prior to GNRs injection (b), 4 min-36 min post injection (c)-(k) in Group I. (1) Experimental timeline for Group I. 


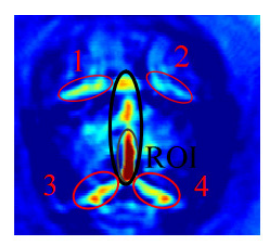

(a)

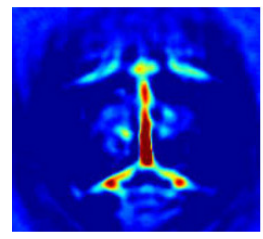

(e)

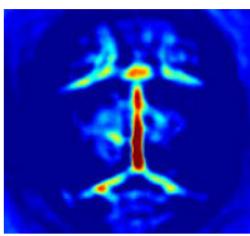

(b)

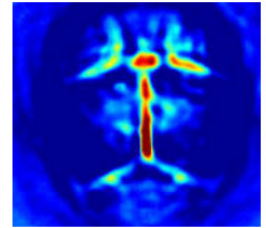

(f)

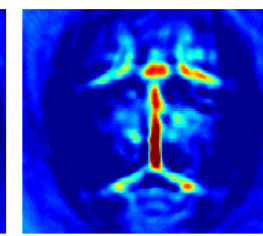

(c)

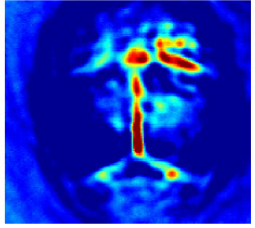

(g)

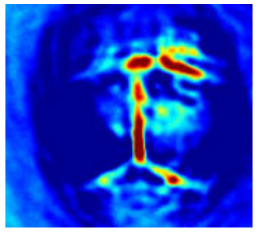

(d)

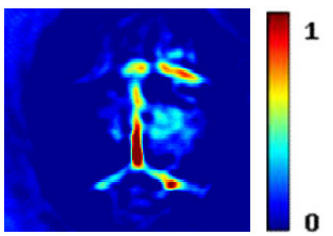

(h)

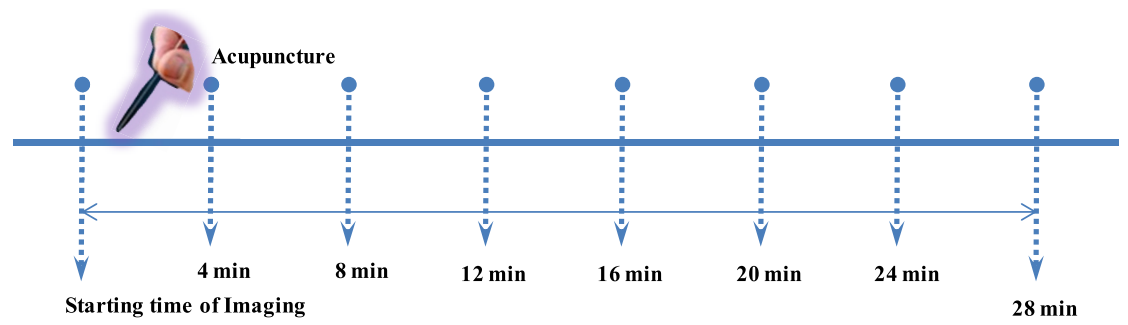

(i)

Fig. 4. (a)-(h) Eight in vivo PAT brain reconstructed images of the cerebral vasculature of a mouse brain prior to acupuncture (a), 4-28 min post acupuncture (b)-(h) in Group II. (i) Experimental timeline for Group II.

of minutely 60 to 70 . As done in the previous studies, two and half minutes after the acupuncture, the needle was retained in the point for more than 28 min. ${ }^{31-34}$ The major blood vessels shown in the PAT image (Fig. 4(a)) were marked by the same anatomical landmarks as Fig. 3(b). After acupuncture, the optical contrast of SSS and the right RRV became noticeably enhanced. The experimental timeline for Group I is shown in Fig. 4(i). Compared to the image prior to injection, the images post injection show some of the brain vasculature with greater clarity. It can be seen that the optical contrast peak of ROI occurred from $8 \mathrm{~min}$ to $12 \mathrm{~min}$ after injection. The absorption decreased dynamically was probably due to the diminishing effect of the acupuncture needle, but it did not fall below the value in the initial state. The results reveal that acupuncture at the ST36 point can promote cerebral hemodynamic parameters, including increasing the concentration of HbT. This is probably due to improved blood supply and reduced cerebral blood vessel resistance, which agrees with the conclusion in the previous study, ${ }^{34}$ that is, acupuncture at the ST36 point could activate some brain areas.
However, not all of the brain areas have increased optical contrast after the mouse was acupunctured. This suggests that acupuncture at the ST36 point cannot activate all of the brain areas. Also, $\mathrm{HbT}$ in the ROI, including SSS in this group was measured in the discussion session.

\subsection{Effect of the combined method on imaging}

Finally, the experiments of Group III $(n=6)$ were used to explore the effect of the optical absorption variation of the vasculature of mouse brain in response to intravenous administration of GNRs and acupuncture at the ST36 point simultaneously. The reconstructed photoacoustic images in Group III are presented in Figs. 5(a)-5(i). As shown in Figs. 5(a)-5(i), photoacoustic images were acquired at night time points for each mouse, i.e., prior to GNRs injection(Fig. 5(a)), 4 min post injection and prior to acupuncture (Fig. 5(b)), and $4 \mathrm{~min}-28 \mathrm{~min}$ post acupuncture (Figs. 5(c)-5(i)). The experimental timeline for Group III is shown in Fig. 5(j). After external stimulus was implemented, we obtained an 
Group III : GNRs+Acupuncture Group

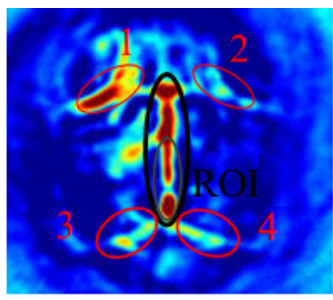

(a)

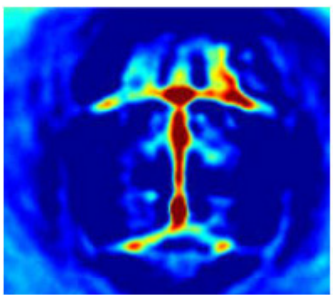

(d)

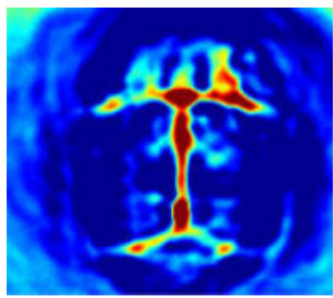

(g)

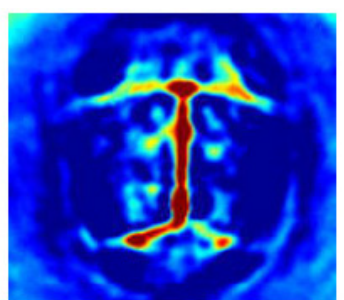

(b)

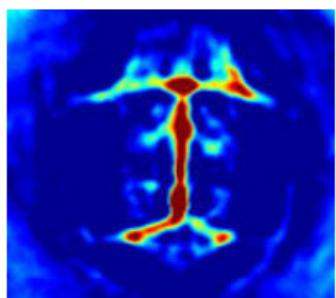

(e)

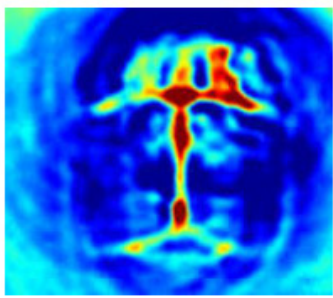

(h)

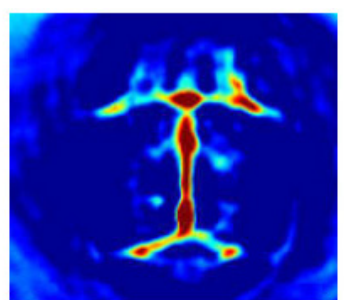

(c)

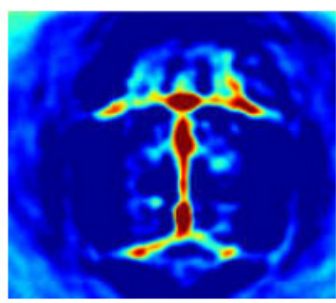

(f)

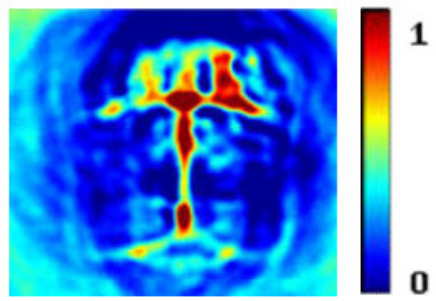

(i)

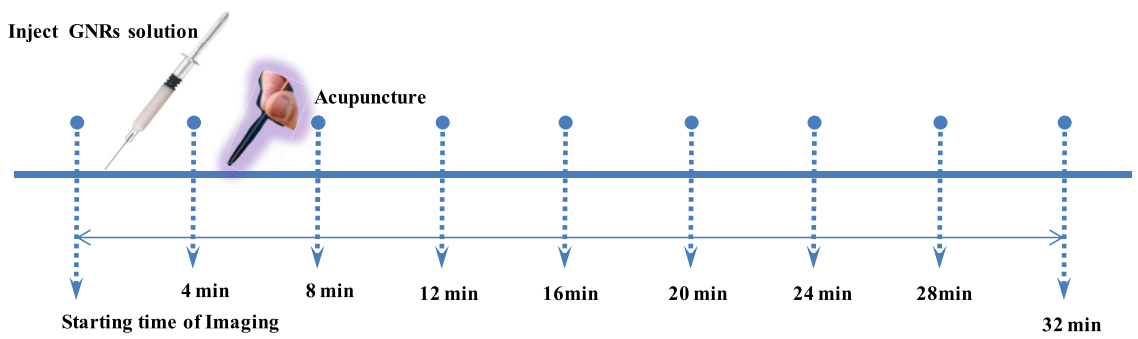

$(\mathrm{j})$

Fig. 5. (a)-(i) Nine in vivo PAT brain reconstructed images of the cerebral vasculature of a mouse brain prior to GNRs injection (a), 4 min post injection and prior to acupuncture (b), and 4 min-28 min post acupuncture (c)-(i) in Group III. (j) Experimental timeline for Group II.

enhanced optical absorption in the in vivo mouse brain images. We imaged the dynamic distribution of NPs in the in vivo mouse brain after acupuncture. Furthermore, it shows there is an optical contrast peak 4 min post injection, and a second peak $8 \mathrm{~min}$ post injection and a third peak $16 \mathrm{~min}$ post acupuncture. The delivery enhancement of NPs or increased concentration of $\mathrm{HbT}$ caused by acupuncture could probably cause the second peak to arise. We need to obtain quantitative data about the relative optical contrast variation to decide the optical absorption variation of the vasculature of mouse brain in response to the integration of acupuncture and contrast agents.

\subsection{Analysis of imaging}

In order to differentiate the optical absorption variation of the vasculature of mouse brain in response to intravenous administration of GNRs and acupuncture at the Zusanli acupoint (ST36) when administered separately and together, we computed 
the quantitative relative optical contrast variation in photoacoustic brain images in the aforementioned three groups of experiments for statistical analysis. We can calculate the pixel value according to the following formula: $\mathrm{SUM}=J \times P$, where $P$ and $J$ is the pixel matrix of the reconstructed image and binary image matrix, respectively. First, the template image of the regions of interest (ROIs) is transformed into the binary image matrix $J$, where regions outside the ROIs were assigned a pixel value of zero. The corresponding position of the binary image was registered with the reconstructed brain image. We then obtained the pixel value, that is, the normalized optical contrast value by multiplying $J$ and $P$. Finally, the normalized optical contrast for the ROIs was calculated. Assuming normalized relative optical contrast is approximately proportional to the $\mathrm{HbT}$ concentration, this measure was used to quantitatively evaluate the effect of the contrast agent and acupuncture. Figure 6 shows a histogram of the percentage variation (SEM) of the normalized relative optical absorption of ROIs over time at intervals of $4 \mathrm{~min}$ for Groups I-III. For Group I, it shows there was an optical contrast peak $(32.08 \pm 12.83 \%) 4 \mathrm{~min}$ post injection, and then a slow downward concussion. For Group II, there was an optical contrast peak $(20.84 \pm 10.87 \%) 8 \mathrm{~min}$ post acupuncture, and then a slow downward concussion. While, we note that when two kinds of exogenous stimulus were applied to the mice in Group III, optical absorption increase presents a slow cyclic oscillation trend. It shows that the 1st optical contrast peak is 4 min post injection, the $2 \mathrm{nd}$ peak at $8 \mathrm{~min}$ post acupuncture and the $3 \mathrm{rd}$ peak is $16 \mathrm{~min}$ post acupuncture (20 min post injection).
We found that the statistical peak induced by acupuncture in Group II was 4 min later than that induced by GNRs injection in Group I. A small but crucial difference between the two groups is that GNRs contrast agent was more effective to stimulate ROI than acupuncture. Besides, it is noted that the value of Group III $(23.05 \pm 9,88 \%)$ was higher than either that of Group I $(18.95 \pm 9.06 \%)$ or that of Group II $(11.99 \pm 4.08 \%) 20 \mathrm{~min}$ post external stimulus. This leads to the conclusion that an integration of two compatible stimulus were more effective to any single stimulus. Importantly, our results also illustrate that an integration of two compatible stimuli could enhance the contrast or increase the concentration of $\mathrm{HbT}$, and activate some areas of the mouse brain. Interestingly, the peak time of the response to acupuncture and administration of GNRs matched each other in the three groups, although the changes of other time points did not correspond to each other. When two kinds of external stimuli were applied simultaneously, their effects were not necessarily linear superposition, and they interacted with each other in an undiscovered way. In order to explore the specific interaction, we need to quantitatively analyze the changes of nanoparticle concentration and hemoglobin concentration in the brain by using multispectral photoacoustic imaging in future research.

Table 1 shows that normalized relative optical contrast variation at one time point in mouse brain for all of the groups. Table 2 shows the statistics of ANOVO in order to assess the changes of optical absorption in different stimulations. ANOVA indicated that the amplitude of SSEPs differed in the

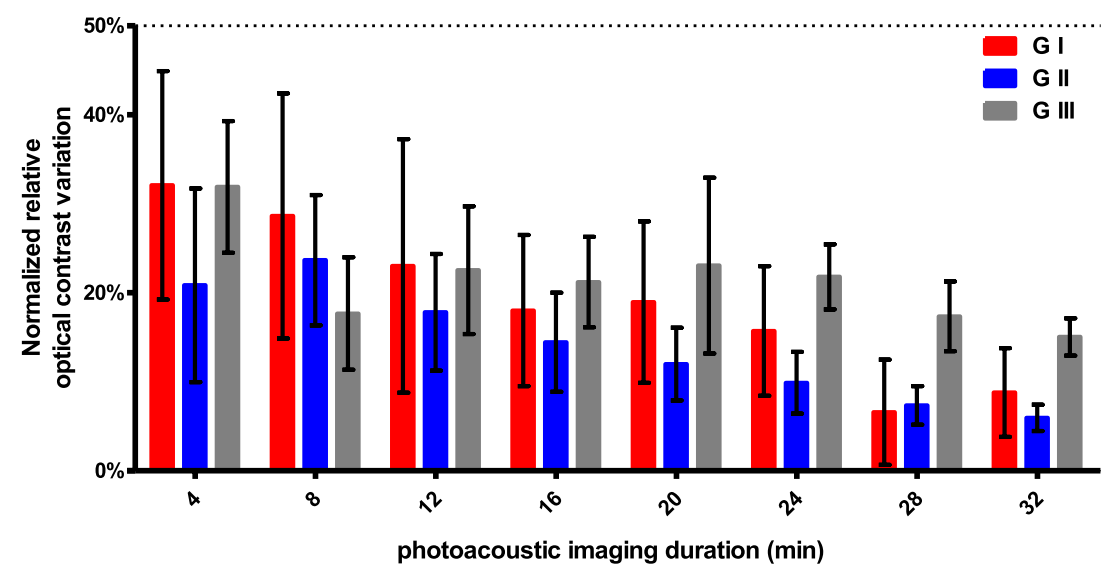

Fig. 6. Histogram of normalized relative optical contrast variation (means \pm SEM) as the timeline for Group I, Group II and Group III. 
Photoacoustic imaging using a composite contrast agent

Table 1. Normalized relative optical contrast variation at one time point in mouse brain for Group I, Group II and Group III.

\begin{tabular}{|c|c|c|c|c|c|c|c|c|c|c|}
\hline \multirow[b]{2}{*}{ Group } & \multicolumn{8}{|c|}{ Normalized relative optical contrast variation at the same time } & \multirow[b]{2}{*}{$n$} & \multirow[b]{2}{*}{ Mean } \\
\hline & No. 1 & No. 2 & No. 3 & No. 4 & No. 5 & No. 6 & No. 7 & No. 8 & & \\
\hline Group I & $10.6 \%$ & $20.5 \%$ & $25.1 \%$ & $37.7 \%$ & $28.7 \%$ & $44.7 \%$ & $47.8 \%$ & $13.9 \%$ & 8 & $28.6 \%$ \\
\hline Group II & $16.4 \%$ & $12.7 \%$ & $10.0 \%$ & $27.7 \%$ & $17.9 \%$ & $21.3 \%$ & - & - & 6 & $17.6 \%$ \\
\hline Group III & $28.6 \%$ & $25.2 \%$ & $11.9 \%$ & $30.2 \%$ & $27.6 \%$ & $30.2 \%$ & $22.3 \%$ & $13.3 \%$ & 8 & $23.7 \%$ \\
\hline
\end{tabular}

Table 2. Statistics of ANOVO.

\begin{tabular}{lrrrrc}
\hline Source of variation & $S$ & $v$ & $M S$ & $F$ & $P$ \\
\hline Total variation & 0.23 & 21 & - & - & - \\
Variation among groups & 0.19 & 2 & 0.095 & 47.5 & $<0.05$ \\
Variation within groups & 0.04 & 19 & 0.02 & - & - \\
\hline
\end{tabular}

three groups $(F=47.5>$ Fcrit(3.52), $P<0.05)$. Therefore, there was significant difference among the experimental groups (Groups I-III). It can be concluded that the effects of GNRs injection, acupuncture at the ST36 point and the combined application of the two have different levels of light absorption in the brains of mice.

\section{Conclusions}

We developed an effective approach of enhancing the photoacoustic imaging contrast, which integrates two key factors, including intravenous injection of an effective contrast agent solution (PEG-GNRs) and a physical auxiliary method (acupuncture at the Zusanli (ST36) point in mice). By employing an appropriate brain functional imaging technology, which was a self-built PAT system in our lab, we successfully captured dynamic change of optical contrast created by PEG-GNRs and induced by acupuncture in the in vivo mouse brain. Furthermore, this study demonstrated that an integration of two compatible stimulus were more effective than any single stimulus. It is revealed that this method can enhance the imaging contrast between the cerebral vessels and the surrounding background tissues and make the vasculature more clear. The above quantitative results also have the potential to estimate the local concentration of GNRs in the brain and even the real-time effects of acupuncture on the brain's functional areas. Acupuncture played a novel role as a contrast agent for photoacoustic imaging in this study. This function is rather different from its conventional one. Beyond that, the use of acupuncture has been developed into an adjunct for symptom control in oncology. Studies with adequate power, duration, and controls have been discovered to evaluate its applications for general symptom management. ${ }^{68}$ Another emerging finding on aupuncture is that a method of acupuncture needle-based plasmonic immunosandwich assay (PISA) has been developed for probing lowcopy-number proteins in living bodies. ${ }^{69}$

Next, we will apply the drug-loaded PEG-GNRs to an in vivo brain disorder model, which will probably integrate traditional acupuncture treatment with pharmacotherapy. The acupuncture will serve not only as a kind of treatment, but also as a physical auxiliary method of drug delivery in the detection and therapy of brain disorders such as cerebral hemorrhage, cerebral thrombosis and cerebral ischemic. Acupuncture combined with drugs has been widely used in the treatment of various clinical conditions and can produce a stronger or more beneficial curative effect than either separate acupuncture or chemical drug does. ${ }^{70-72}$ However, the mechanisms underlying the additive or synergetic effects of acupuncture plus drugs are not well understood so far. Cui et al., proposed several aspects possibly involving the additive or synergetic effects produced by acupuncture plus drugs. ${ }^{73}$ For instance, acupuncture stimulation at acupoints may influence the drug concentration in blood and/or in target organ and the bioavailability of the drug via modulating the drug absorption, distribution and metabolism in the body. ${ }^{74-76}$ In addition, the curative effect of drug may also be strengthened by acupuncture stimulation via altering the signaling pathways of the targeted specific receptors and via potentiating the resultant responsiveness or sensitivity of the cells in the target organ in responding to the drugs administered. ${ }^{77-84}$ The ideas mentioned above may raise the novel clues for further studying the mechanisms underlying acupuncture-produced increase of the curative effects of medicines. We should further the study to show the interactions 
between specific acupuncture points and contrast agents using multispectral photoacoustic imaging, which will help discover new methods to promote the delivery and distribution of contrast agents and drugs.

\section{Acknowledgment}

The authors are grateful to Y. Tang from CHENGDU University of TCM for theory support of traditional Chinese medicine.

\section{References}

1. A. G. Bell, "On the production and reproduction of sound by light," Am. J. Sci. 20, 305-324 (1880).

2. P. Beard, "Biomedical photoacoustic imaging," Interface Focus 1, 602-631 (2011).

3. J. Tang, L. Xi, J. Zhou, H. Huang, T. Zhang, P. Carney, H. Jiang, "Noninvasive high-speed photoacoustic tomography of cerebral hemodynamicsin awake-moving rats," J. Cereb. Blood Flow Metab. 35, 1224-1232 (2015).

4. D. Wu, L. Huang, M. S. Jiang, H. Jiang, "Contrast agents for photoacoustic and thermoacoustic imaging: A review," Int. J. Mol. Sci. 15, 23616-23639 (2014).

5. A. Taruttis, S. Morscher, N. C. Burton, D. Razansky, V. Ntziachristos, "Fast multispectral optoacoustic tomography (MSOT) for dynamic imaging of pharmacokinetics and biodistribution in multiple organs," Plos One 7, e30491 (2012).

6. H. Jiang, Photoacoustic Tomography, CRC Press (2014).

7. L. Xi, S. R. Grobmyer, L. Wu, R. Chen, G. Zhou, L. G. Gutwein, J. Sun, W. Liao, Q. Zhou, H. Xie, H. Jiang, "Evaluation of breast tumor margins in vivo with intraoperative photoacoustic imaging," Opt. Express 20, 8726-8731 (2012).

8. M. Nasiriavanaki, J. Xia, H. Wan, A. Q. Bauer, J. P. Culver, L. V. Wang, "High-resolution photoacoustic tomography of resting-state functional connectivity in the mouse brain," Proc. Natl. Acad. Sci. 111, 21 (2014).

9. D. Wu, J. Yang, G. Zhang, H. Jiang, "Noninvasive in vivo monitoring of collagenase induced intracerebral hemorrhage by photoacoustic tomography," Biomed. Opt. Exp. 8, 2276-2286 (2017).

10. D. Wu, X. Guo, R. Cui, M. Wu, Q. Shang, H. Jiang, "In vivo hemodynamic visualization of berberineinduced effect on the cerebral cortex of a mouse by photoacoustic tomography," Appl. Opt. 58, 1-8 (2019).

11. P. Zhang, L. Li, L. Lin, P. Hu, J. Shi, Y. He, L. Zhu, Y. Zhou, L. V. Wang, "High resolution deep functional imaging of the whole mouse brain by photoacoustic computed tomography in vivo," $J$. Biophoton. 11, e201700024 (2018).

12. S. Liu et al., "Electromagnetic-acoustic sensing for biomedical applications," Sensors 18(10), 3203 (2018).

13. D. Wang, Y. Wu, J. Xia, "Review on photoacoustic imaging of the brain using nanoprobes," Neurophotonics 3(1), 010901 (2016).

14. L. Nie, Z. Guo, L. V. Wang, "Photoacoustic tomography of monkey brain using virtual point ultrasonic transducers," J. Biomed. Opt. 16(7), 076005 (2011).

15. X. Wang, D. L. Chamberland, G. Xi, "Noninvasive reflection mode photoacoustic imaging through infant skull toward imaging of neonatal brains," J. Neurosci. Meth. 168(2), 412-421 (2008).

16. X. Wang et al., "Reflection mode photoacoustic imaging through infant skull toward noninvasive imaging of neonatal brains," J. Neurosci. Meth. 168(2), 412-421 (2009).

17. A. Hariri et al., "Functional photoacoustic tomography for neonatal brain imaging: Developments and challenges," Proc. Photons Plus Ultrasound: Imaging 83 Sensing. International Society for Optics and Photonics, p. $10642 \mathrm{Z}$ (2017).

18. S. Herrmann et al., "Cerebral blood oxygenation measurements in neonates with optoacoustic technique," Soc. Photo-Opt. Instrum. Eng. (SPIE) Conf. Series, p. 100640Q (2017).

19. C. Huang, "Aberration correction for transcranial photoacoustic tomography of primates employing adjunct image data," J. Biomed. Opt. 17(6), 066016 (2012).

20. A. R. Mohammadi-Nejad et al., "Neonatal brain resting-state functional connectivity imaging modalities," Photoacoustics S2213597917300368 (2018).

21. K. Kim et al., "Photoacoustic imaging of early inflammatory response using gold nanorods," Appl. Phys. Lett. 90(22), 223901 (2007).

22. H. W. Yang et al., "Magnetic gold-nanorod/PNIPAAmMA nanoparticles for dual magnetic resonance and photoacoustic imaging and targeted photothermal therapy," Biomaterials 34, 5651-5660 (2013).

23. P. H. Wang et al., "Gold-nanorod contrast-enhanced photoacoustic micro-imaging of focused-ultrasound induced blood-brain-barrier opening in a rat model," J. Biomed. Opt. 17(6), 061222 (2012).

24. P. C. Li et al., "In vivo photoacoustic molecular imaging with simultaneous multiple selective targeting using antibody-conjugated gold nanorods," Opt. Exp. 16, 18605-18615 (2008).

25. J. E. Millstone et al., "Observation of a quadrupole plasmon mode for a colloidal solution of gold nanoprisms," J. Am. Chem. Soc. 127, 5312-5313 (2005). 
26. P. K. Jain et al., "Calculated absorption and scattering properties of gold nanoparticles of different size, shape, and composition: Applications in biological imaging and biomedicine," J. Phys. Chem. B 110, 7238-7248 (2006).

27. C. M. Chuang et al., "Acupuncture stimulation at Baihui acupoint reduced cerebral infarct and increased dopamine levels in chronic cerebral hypoperfusion and ischemia-reperfusion injured SpragueDawley rats," Am. J. Chin. Med. 35(5), 779-791 (2007).

28. Y. F. Qian et al., "Effects of acupuncture at different acupoints on cerebral blood flow in cerebral ischemia model rats," Zhongguo Zhenjiu 29(3), 213216 (2009).

29. H. $\mathrm{Xu}$ et al., "Effects of acupuncture at Baihui (DU20) and Zusanli (ST36) on the expression of heat shock protein 70 and tumor necrosis factor $\alpha$ in the peripheral serum of cerebral ischemia-reperfusioninjured rats," Chin. J. Integr. Med. 20(5), 369-374 (2014).

30. B. Zhu, Scientific Foundations of Acupuncture $\&$ Moxibustion, Qingdao Press (1998).

31. B. Z. Chen et al., "Photoacoustic imaging of cerebral hypoperfusion during acupuncture," Biomed. Opt. Exp. 6(9), 3225-3234 (2015).

32. T. Li et al., "Photoacoustic imaging of acupuncture effect in small animals," Biomed. Opt. Exp. 6(2), 433-442 (2015).

33. J. Yang et al., "Photoacoustic microscopy of electronic acupuncture (EA) effect in small animals," J. Biophoton. 10(2), 217 (2016).

34. W. Dan, H. Jiang, "Contrast enhanced photoacoustic tomograpgy of living mouse brain using combined acupuncture and contrast agents," Acta Laser Biol. Sin. 27(2), 133-141 (2018).

35. G. Li et al., "Cortical activations upon stimulation of the sensorimotor-implicated acupoints," Magn. Reson. Imaging. 22(5), 639-644 (2004).

36. D. Alimi, A. Geissmann, D. Gardeur, "Functional MRI of the human brain following auricular stimulation," Med. Acupuncture. 13(2), 64 (2009).

37. C. M. Siedentopf et al., "Functional magnetic resonance imaging detects activation of the visual association cortex during laser acupuncture of the foot in humans," Neurosci. Lett. 327(1), 53-56 (2002).

38. S. S. Yoo et al., "Modulation of cerebellar activities by acupuncture stimulation: Evidence from fMRI study," Neuroimage 22(2), 932-940 (2004).

39. Z. H. Cho et al., "New findings of the correlation between acupoints and corresponding brain cortices using functional MRI," Proc. Natl. Acad. Sci. 95(5), 2670-2673 (1998).

40. W. T. Zhang et al., "Evidence from brain imaging with fMRI supporting functional specificity of acupoints in humans," Neurosci. Lett. 354(1), 50-53 (2004).

41. Y. Shifang, N. Mofan, W. Ling, "Hemodynamics effect of negative pressure on ischemic extremity," China J. Mod. Med. 11, 010 (2003).

42. H. Maeda, H. Nakamura, J. Fang, "The EPR effect for macromolecular drug delivery to solid tumors: Improvement of tumor uptake, lowering of systemic toxicity, and distinct tumor imaging in vivo," $A d v$. Drug Deliv. Rev. 65(1), 71-79 (2013).

43. H. Jiang et al., "Related method of selective opening blood brain barrier," Med. Recapitulate 11, 023 (2010).

44. K. Linnet, T. B. Ejsing, "A review on the impact of P-glycoprotein on the penetration of drugs into the brain. Focus on psychotropic drugs," Eur. Neuropsychopharmacol 18(3), 157-169 (2008).

45. M. A. Deli, "Potential use of tight junction modulators to reversibly open membranous barriers and improve drug delivery," Biochim. Biophys. Acta 1788(4), 892-910 (2009).

46. E. Preston et al., "Graded reversible opening of the rat blood-brain barrier by intracarotid infusion of sodium caprate," J. Neurosci. Meth. 168(2), 443-449 (2008).

47. R. Singh, J. W. Lillard Jr., "Nanoparticle-based targeted drug delivery," Exp. Mol. Pathol. 86(3), 215-223 (2009).

48. L. Liu et al., "Biologically active core/shell nanoparticles self-assembled from cholesterol-terminated PEG-TAT for drug delivery across the blood-brain barrier," Biomaterials 29(10), 1509-1517 (2008).

49. F. Y. Yang, P. Y. Lee, "Efficiency of drug delivery enhanced by acoustic pressure during blood-brain barrier disruption induced by focused ultrasound," Int. J. Nanomed. 7, 2573-2582 (2012).

50. Y. C. Kuo, C. Y. Kuo, "Electromagnetic interference in the permeability of saquinavir across the blood-brain barrier using nanoparticulate carriers," Int. J. Pharm. 351(1/2), 271-281 (2008).

51. Y. Wu, G. L. Liu, "Radiotherpy-induced Bloodbrain Barrier disruption and its implication for chemotherapy," Chin. J. Neuro-Oncol. 5(1), 63-65 (2007).

52. K. P. Tan, X. M. Lin, "Research and development tendency on Blood-brain Barrier with Chinese Herbs and acupuncture," Chin. Arch. Traditional Chin. Med. 25(11), 2283-2285 (2007).

53. J. Chen et al., "Gold nanocages: Bioconjugation and their potential use as optical imaging contrast agents," Nano Lett. 5(3), 473-477 (2005).

54. W. C. W. Chan et al., "Luminescent quantum dots for multiplexed biological detection and imaging," Curr. Opin. Biotechnol. 13(1), 40-46 (2002). 
55. X. Gao et al., "In vivo molecular and cellular imaging with quantum dots," Curr. Opin. Biotechnol. 16(1), 63-72 (2005).

56. X. Feng, F. Gao, Y. Zheng, "Thermally modulated photoacoustic imaging with super-paramagnetic iron oxide nanoparticles," Opt. Lett. 39(12), 3414-3417 (2014).

57. Y. Jin et al., "Multifunctional nanoparticles as coupled contrast agents," Nat. Commun. 1, 41 (2010).

58. A. De La Zerda et al., "Carbon nanotubes as photoacoustic molecular imaging agents in living mice," Nat. Nanotechnol. 3(9), 557-562 (2008).

59. S. Zanganeh et al., "Photoacoustic imaging enhanced by indocyanine green-conjugated single-wall carbon nanotubes," J. Biomed. Opt. 18(9), 096006 (2013).

60. P. K. Avti et al., "Detection, mapping, and quantification of single walled carbon nanotubes in histological specimens with photoacoustic microscopy," PLoS One 7(4), e35064 (2012).

61. V.P. Nguyen et al., "Enhancement of high-resolution photoacoustic imaging with indocyanine greenconjugated carbon nanotubes," Jpn. J. Appl. Phys. 54(7S1), 07HF04 (2015).

62. S. K. Maji et al., "Upconversion nanoparticles as a contrast agent for photoacoustic imaging in live mice," Adv. Mater. 26(32), 5633-5638 (2014).

63. J. Chen et al., "Gold nanocages: Engineering their structure for biomedical applications," Adv. Mater. 17(18), 2255-2261 (2005).

64. Y. Wang et al., "Photoacoustic tomography of a nanoshell contrast agent in the in vivo rat brain," Nano Lett. 4(9), 1689-1692 (2004).

65. W. Lu et al., "Photoacoustic imaging of living mouse brain vasculature using hollow gold nanospheres," Biomaterials $\mathbf{3 1}(9)$, 2617-2626 (2010).

66. G. $\mathrm{Xu}$ et al., "Theranostic quantum dots for crossing blood-brain barrier in vitro and providing therapy of HIV-associated encephalopathy," Front. Pharmacol. 4, 1-8 (2013).

67. S. Hanada et al., "Cell-based in vitro blood-brain barrier model can rapidly evaluate nanoparticles' brain permeability in association with particle size and surface modification," Int. J. Mol. Sci. 15(2), 1812-1825 (2014).

68. M. K. Garcia et al., "Systematic review of acupuncture in cancer care: A synthesis of the evidence," J. Clin. Oncol. 31(7), 952-960 (2013).

69. J. Liu et al., "Probing low-copy-number proteins in a single living cell," Angewandte Chemie Int. Ed. 55(42), 13215-13218 (2016).

70. W. Zhu, G. Xi, J. Ju, "Effect of acupuncture and Chinese medicine treatment on brain dopamine level of MPTP-lesioned C57BL mice," Acupuncture Res. 21(4), 46 (1996).
71. J. Chen, "Influence of acupuncture on serum growth hormone level in patients with type II diabetes," Acupuncture Res. 26(4), 310-313 (2001).

72. Y. Shi et al., "Effects of acupuncture combined with medicine on the expression of IFN- $\gamma$ and IL-12 of colonic mucous membrane in ulcerative colitis rats," Acupuncture Res. 30(4), 215-218 (2005).

73. J. J. Cui et al., "Discussion on the novel clues for studying the underlying mechanisms of acupunctureinduced potentiation of the curative effect of medicines," Acupuncture Res. 35(2), 146-150 (2010).

74. Y. Chen, W. Jia, Z. Cheng, "Effects of electroacupuncture on tropism of effective component of pubescent angelica root in the rat with adjuvant arthritis," Chin. Acupuncture Moxibust. 24(1), 5961 (2004).

75. Y. H. Gu, H. Z. Jin, S. D. Li, "The influence of acupuncture on the intracorporeal metabolism of tripterygium wilfordii in rate with adjuvant arthritis," Shanghai J. Acupuncture Moxibust. 20(5), 41-43 (2001).

76. S. Zhang, H. Niu, "The effect of acupoint injection of capsaicin on the neurogenic inflammation induced by primary afferent reflexs," J. Xian Med. Univ. 21(1), 6-8 (2000).

77. Z. Cheng, Y. Chen, L. Zhang, "Experimental study on effect of acupuncture on tropism of effective compositions of the Chinese drug in the rat," Chin. Acupuncture Moxibust. 22(1), 51-53 (2002).

78. T. Zhang, C. Gao, Y. Guo, "Effects of moxibustion on the function of MDR gene product, P-glycoprotein (P-170)," Acupuncture Res. 19(2), 69-71 (1994).

79. E. Pinter, J. Szolcsanyi, "Plasma extravasation in the skin and pelvic organs evoked by antidromic stimulation of the lumbosacral dorsal roots of the rat," Neurosci. 68(2), 603-614 (1995).

80. J. Fang et al., "Neurogenic inflammation evoked by stimulation of the acupoint through drr," J. Xian Med. Univ. 1999(2), 175 (1999).

81. D. Cao, H. Niu, Z. Yan, "Neurogenic inflammation of the visceral organs evoked by electrical stimulation of acupoint in rats," Chin. Acupuncture Moxibust. 21(11), 662-664 (2001).

82. S. Zhang, H. Niu, S. Jiang, "Nerogenic inflammation evoked by stimulation of acupoint through long axon reflex," J. Xian Med. Univ. 20(4), 438-440 (1999).

83. X. C. Yu et al., "Cross-talk between cardiac kappaopioid and beta-adrenergic receptors in developing hypertensive rats," J. Mol. Cell. Cardiol. 31(3), 597 (1999).

84. J. Gao et al., "Acupuncture pretreatment protects heart from injury in rats with myocardial ischemia and reperfusion via inhibition of the $\beta$-adrenoceptor signaling pathway," Life Sci. 80(16), 1484-1489 